\title{
Electric Circuit Analysis in MATLAB and Simulink
}

\section{Dr. Asad Yousuf, Savannah State University \\ Dr. Mohamad A. Mustafa, Savannah State University \\ Mr. William Lehman, Bill's Robotic Solutions}

William Lehman is President of Bill's Robotic Solutions which he started in July of 2013. He has had over twenty years of experience in software and hardware development. He has worked on numerous projects in digital communication systems, robotics, and aerospace applications. For the past seven years he has taught technology education mainly at the high school level. Mr. Lehman received his Bachelor of Science degree in Electrical Engineering in 1979 from Catholic University of America, Washington DC. 


\title{
Electric Circuit Analysis in MATLAB and Simulink
}

\begin{abstract}
Electric Circuit Analysis I is the first course that the students take in Electrical Engineering Technology and the dropout rate is high in this course because students lose interest in just solving problems and analyzing them using simulation software packages. The predesigned software packages are not helpful in understanding the calculation and analysis of electrical circuit components. This paper will discuss Electrical Circuit Analysis in MATLAB and Simulink. Electrical Circuit analysis activity demands an interdisciplinary approach which promotes collaborative project-based learning (PBL). During the undergraduate summer research training program the students were given the task to solve problems in Electric Circuit I course. However, the students working on this had the Electric Circuit I course during the academic year. These students were selected as tutors for the students taking the Electric Circuit I course in the fall semester to help them understand the concept of Electric Circuit I problem solving using MATLAB. It was observed that with implementing the problems in MATLAB the students were gaining a better understanding of Electric Circuit problems and their interest level was also increased which resulted in better retention in the course. The name MATLAB stands for MATrix LABoratory. MATLAB was written originally to provide easy access to matrix software developed by the LINPACK (linear system package) and EISPACK (Eigen system package) projects. MATLAB is computational in nature which provides conceptual approach for designing and solving problems in Electrical Circuits. MATLAB has embedded software called SIMULINK which provides an essential way to model, simulate and analyze Electrical Systems which are characterized by some inputs and outputs. This paper will discuss the summer undergraduate research training project in which the students tested the basic electrical circuits to explore Basic DC/AC circuit computations. The students were also introduced to design/implementation, testing and verification. Students not only worked with other students taking the Electric Circuit I course on campus during fall semester but also worked with the area high school students during the summer programs conducted for creating interest in Electrical Engineering Technology programs.
\end{abstract}

\section{Introduction}

Students enrolled in Electronics and Civil Engineering Technology are required to take a fourcredit hour Electrical Circuit I course. The only pre-requisite for the course is pre-calculus, and the course is designed to inspire interest among students and introduce the students to the concepts of electrical circuits in freshman year. Freshman retention has been a critical issue for Electronics Engineering Technology programs over the last decade. Some State Universities have implemented many different approaches to improve the retention of freshmen. Some of these attempts include: creating basic Electronics Engineering Technology courses such as Electrical Circuit I in freshman year to give early hands-on experience to the students, and moving the ownership and maintenance of laboratory equipment from the university to the students. Introduction of Electrical Circuits I course in the freshman year of Electrical Engineering Technology program will enhance interest in Electrical Engineering Technology leading to higher matriculation rates, increased retention, and ultimately a higher graduation rate. 
Electrical Circuits I course serves as a gateway to all Electrical Engineering Technology curriculums across the globe. The purpose of the course is to introduce the students' to the concepts of passive elements and the associated laws that direct the behavior of the circuit when the elements are connected in a circuit or a system. The circuits sequence course which consists of Electrical Circuit I (DC concepts) and Electrical Circuit II (AC concepts) had been conducted in traditional format which includes problem solving, conducting lab experiments and performing the calculation with TI-89 or other standard calculator. This training methodology may seem to be effective means of delivering information while the students are registered in the course. However, many faculty members expressed the opinion that the students in the upper division courses did not retain the knowledge of basic circuit theory that they expected. Perhaps, if students were engaged in programming the basic concepts such as, Series Circuit, Series/Parallel circuits, Nodal Analysis, Mesh Analysis, Maximum Power Theorem, Thevenin's Theorem, Norton Theorem, and Superposition Theorem, using MATLAB they may retain circuit analysis information better since they have to really understand the circuit concepts in order to program in MATLAB. Furthermore, the students in today's technological world do not rely on college instructor's lecture note but prefer to verify the theoretical material through the use of computer system and technology. We can capitalize on this trend of interest of our students in computer to promote learning which will make them more interested in the material that is being covered in the classroom. There are numerous numbers of software packages in the market such as Multisim, PSPICE etc. which are excellent for the students to learn about simulation of circuits. However, deeper understanding of the circuits can be promoted if students were writing their own code in environment like MATLAB and then simulate the circuit in Simulink to analyze the circuit behavior. This paper will discuss the summer undergraduate research training project in which the students analyzed electrical circuits in MATLAB and Simulink platform. The paper describes the following:

- Overview of MATLAB, Simulink and Electrical Circuits

- Electrical Circuit Problem Solving using MATLAB and Simulink

- Student retention and Outreach

\section{Overview of MATLAB, Simulink and Electrical Circuits:}

MATLAB (Matrix Laboratory) as a computational software is receiving phenomenal attention from engineering and scientific communities. MATLAB avoids the awkward compilation often encountered in traditional languages such as C++, JAVA etc. Furthermore it provides an opportunity for easy debugging, easy to execute workspace, and uses the built in library functions which avoids hundreds of programming statements. Also, provides the convenience of Graphical User Interface (GUI) design tools as well as scientific modelling platform without laborious programming. Simulink as an additional part of MATLAB has enhanced the application of the software package. MATLAB is based on matrices whereas SIMULINK is composed of blocks.

Simulink is integrated with MATLAB which is used for modelling, simulating and analyzing of dynamic systems and also provides support for linear and nonlinear systems which are modelled in continuous time, sampled time or a cross of two systems. Simulink turns your computer into a laboratory environment for modelling and analyzing systems such as electrical engineering systems. Electrical circuit calculation using a traditional calculator is almost becoming a thing of 
the past. Students need to focus on understanding the concept behind an electric circuit instead of just plugging in numbers in a calculator. Electrical Circuits I is designed to introduce the basic concepts in DC circuits, simulation and DC circuit labs, followed by Electrical Circuits II, which covers the advanced topics in AC, simulation and AC circuit labs.

Following topics are covered in Electrical Circuits I and II sequence courses:

1. Voltage and Current

2. Resistance

3. Ohm's Law, Power, and Energy

4. Series DC circuits

5. Parallel DC circuits

6. Network Theorems

7. Mesh and Nodal Analysis

8. Sinusoidal Alternating Waveforms

9. The Basic Elements and Phasors

10. Series and Parallel AC circuits

11. Network theorems AC circuits

12. Power and Resonance

13. Transformers

14. Polyphase systems

The study of topics 1-7 occurs in Electrical Circuits I, with the remaining topics studied the following semester in Electrical Circuits II. Traditionally, the Electric Circuit I course was based on lecture and lab experiments which were provided in the format of laboratory manual by the author of the textbook. In Fall 2013 the course was revised based on the concept of introducing a software lab exercise in each class period in which the concept of programming electrical circuits problems using MATLAB was introduced. In addition, a team of four students was asked to work on lab exercises outside the classroom. Summer undergraduate research training students who worked on DC/AC circuit computation using MATLAB provided a helping hand to the students enrolled in section 1 of Electric Circuit I. There were two sections of Electric Circuit I offered in Fall 2013. Both the sections used the same textbook and the topics covered were the same. In section 1 students- were introduced to MATLAB and were required to write MATLAB code for the concepts learned during the class period. The other class was the control group in which the students were taught in the traditional manner. The introduction of MATLAB was supported by the traditional contextual teaching philosophies based on the following principles:

- Work-based learning (an approach in which workplace, or workplace-like, activities are integrated with classroom content for the benefit of students.)

- Problem-based learning (an approach that engages learners in problem-solving investigations that integrate skills and concepts from many content areas.)

- Cooperative learning (an approach that organizes instruction using small learning groups in which students work together to achieve learning goals)

- Project-based learning (an approach that focuses on the central concepts and principles of a discipline, involves students in problem-solving investigations) 
Following paragraphs will discuss the Electric Circuit Problem Solving using MATLAB and Simulink, student retention in Electric Circuit I course and student outreach.

\section{Electrical Circuit Problem Solving using MATLAB and Simulink:}

During the summer undergraduate research program students explored the application of MATLAB and Simulink to solve Electrical Circuit I problems. With this foundation the undergraduate research students were able to provide assistance with MATLAB and Simulink to the students who were taking the Electrical Circuit I during the fall semester of 2013. Following paragraphs will discuss the following:

- Basic DC circuit Computations

- DC Circuit Modeling

\section{Basic DC Circuit Computations}

Basic circuit computation is needed in electrical circuit problem solving. Students wrote functions which were not available in MATLAB and the use of which seems very useful in solving electrical circuit problems. An example of such computation is finding the equivalent resistance in a circuit. This function once built can be used repeatedly in solving circuit problems. A master equation solver in MATLAB can find the solution of single or simultaneous linear algebraic or trigonometric equations. The following paragraphs will discuss the function of Series-Parallel Circuit Resistance computation and DC Nodal analysis computation by using the built-in circuit equation solver.

\section{Series-Parallel Circuit Resistance Computation}

In a given electrical circuit the basic circuit computation begins with the equivalent resistance computation of a series parallel network. There is no built in function in MATLAB for this type of computation. Figure 1.0 below shows two resistors connected in parallel which will be modeled as a function in MATLAB and can be used as the basis for computing equivalent resistance of the series parallel circuit.

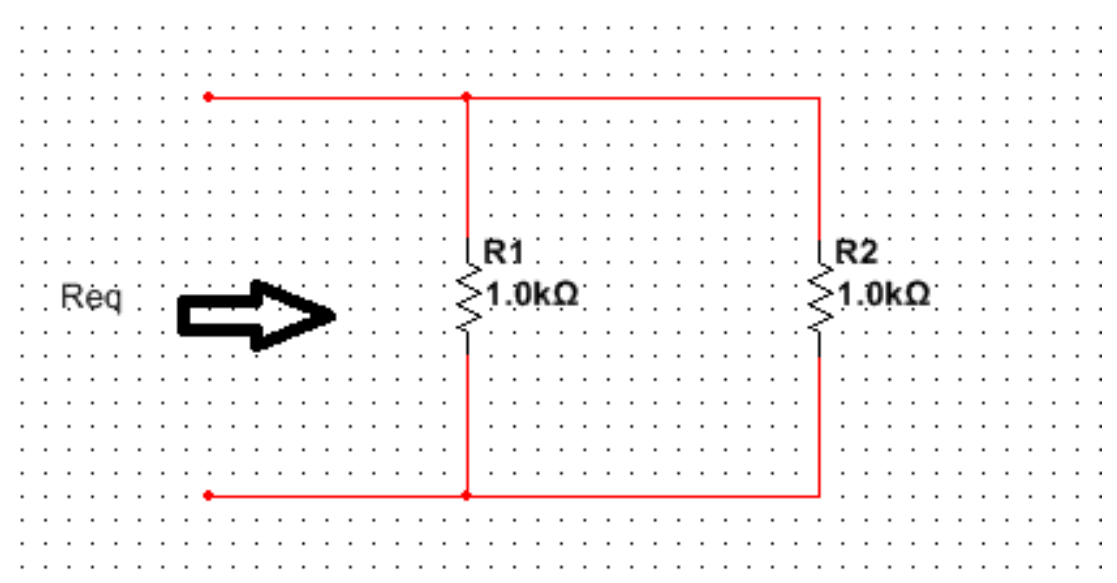

Figure 1.0: Two resistors in Parallel 
MATLAB functions are similar in nature to $\mathrm{C}++$ or traditional programming languages. The MATLAB functions can be used interactively. In addition to the interactive calculation, MATLAB also supports an easier way to support debug functions that are part of longer programs. MATLAB have two parameter lists, one for input and one for output. The first line of a function $\mathrm{m}$-file is in the following format:

\section{Function[output_parameters]=function_name(input_parameters)}

The first word is always the word function which is followed by the optional output parameters enclosed in the square brackets. If the function has no output parameters the square brackets and the equal sign can be omitted. File saved must have the same as the function name and the function name is a character string that will be used to call the function. The equivalent resistance of figure 1.0 is given by the equation below:

$$
R e q=\frac{R 1 R 2}{R 1+R 2}
$$

Following are steps to write a function called parallel:

1. Under file menu in MATLAB click new function.

2. Write the function with the following lines:

Function Rtotal=parallel( $R 1, R 2)$

Rtotal $=(R 1 * R 2) /(R 1+R 2)$;

End

Once we have our function developed then we can use the base function to find total resistance of series/parallel network. We can compute the total resistance of the network in figure 2.0 by invoking the function at the command prompt with the following sequence:

> $R 1=4 ; R 2=6 ; R 3=6 ; R 4=6 ;$
$>>$ total $=R 1+\operatorname{Parallel}(\operatorname{Parallel}(R 2, R 3), R 4)$

Rtotal $=6$

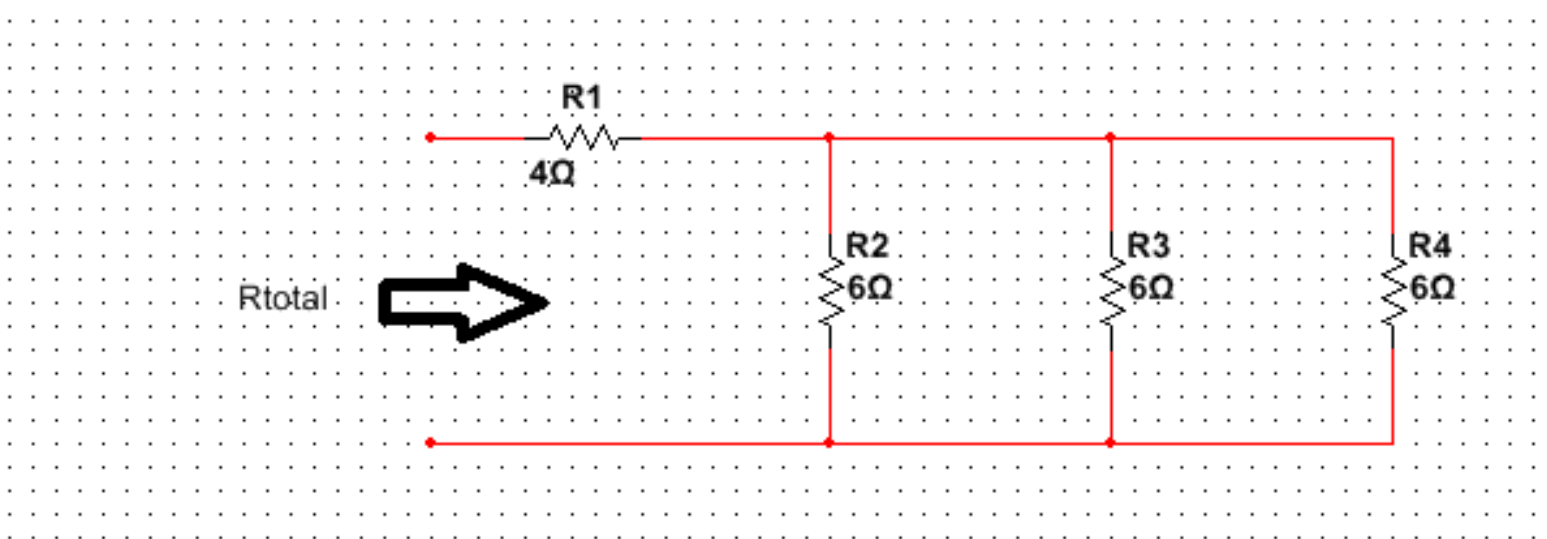

Figure 2.0 Three Parallel resistors $(\mathrm{R} 2, \mathrm{R} 3, \mathrm{R} 4)$ in series with R1 


\section{Nodal Analysis Computation:}

Nodal analysis provides a general procedure for analyzing circuits using node voltage as the circuit variables. The nodes of the circuit are the places where they are connected together. The circuit shown in figure 3.0 has three nodes. Node 3 is the reference node or the datum node. Analyzing the connected circuit containing n nodes will require $\mathrm{n}-1 \mathrm{KCL}$ equations. The KCL equations are obtained from each node with exception of the reference node or the datum node. Following are steps to determine the nodal voltages:

- Express element current as function of the nodal voltage

- Apply KCL to each node with the exception of reference node

- Solve the resulting simultaneous equations to obtain the unknown voltages.

The resulting simultaneous can be solved efficiently and accurately by using the circuit equation solver or we can use matrix method in MATLAB. The KCL equations can also be solved by using a calculator but this method is not as efficient as the MATLAB.

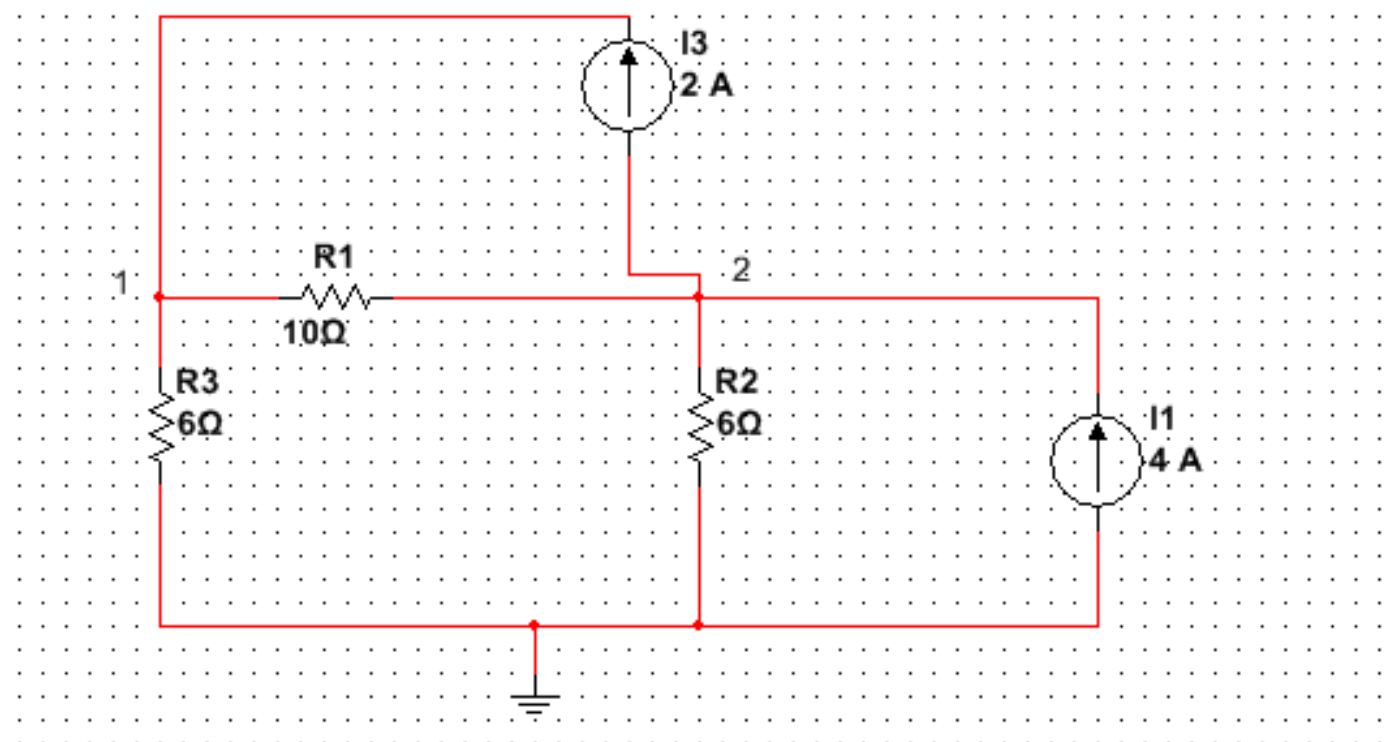

Figure 3.0

Applying KCL to node 1 of circuit in figure 3.0 we can obtain the following equations:

$$
\begin{aligned}
& i 1=i 2+i 3 \\
& 2=\frac{v 1-0}{6}+\frac{v 1-v 2}{10}
\end{aligned}
$$

Applying KCL to node 2 of circuit in figure 3.0 we can obtain the following equations:

$$
i 2+i 4=i 1+i 5
$$




$$
\frac{v 1-v 2}{10}+4=2+\frac{v 2-0}{6}
$$

We can compute v1 and v2 by following two methods in MATLAB:

- Circuit Equation Solver

- Matrix Method

\section{Circuit Equation Solver:}

| By using the built-in master function solver, we can find the solution of a-single, multiple, algebraic equations when the equations are its input arguments. The format of the solution is symbolic in nature and a substantial number of simultaneous linear, algebraic or trigonometric equations can be solved by using the solve function. Below is the MATLAB code at the command prompt to solve for $\mathrm{v} 1$ and $\mathrm{v} 2$ :

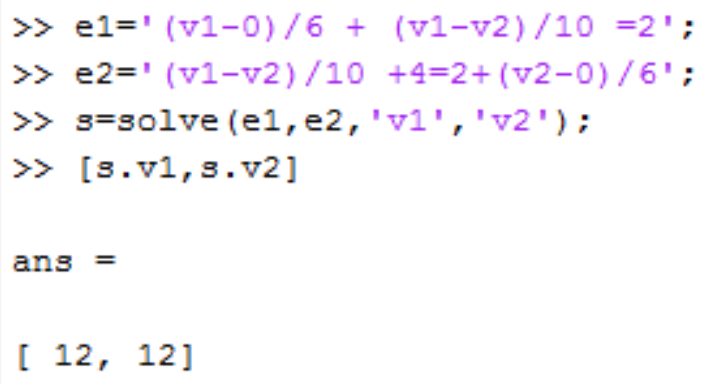

\section{Matrix Method:}

We can also use the matrix method to solve for $v 1$ and v2. To solve for v1 and v2 using the Cramer's rule or matrix inversion we have to represent equations 3 and 5 in the following format:

$$
\begin{aligned}
& \left(\frac{1}{6}+\frac{1}{10}\right) v 1-\frac{v 2}{10}=2 \\
& \frac{v 1}{10}-v 2\left(\frac{1}{10}+\frac{1}{6}\right)=-2
\end{aligned}
$$

Equations (6) and (7) can be represented in the matrix format in the following manner:

$$
\left[\begin{array}{cc}
\frac{1}{6}+\frac{1}{10} & -\frac{1}{10} \\
\frac{1}{10} & -\left(\frac{1}{10}+\frac{1}{6}\right)
\end{array}\right] *\left[\begin{array}{l}
v 1 \\
v 2
\end{array}\right]=\left[\begin{array}{c}
2 \\
-2
\end{array}\right]
$$


Below is the MATLAB code at the command prompt to solve for v1 and v2:

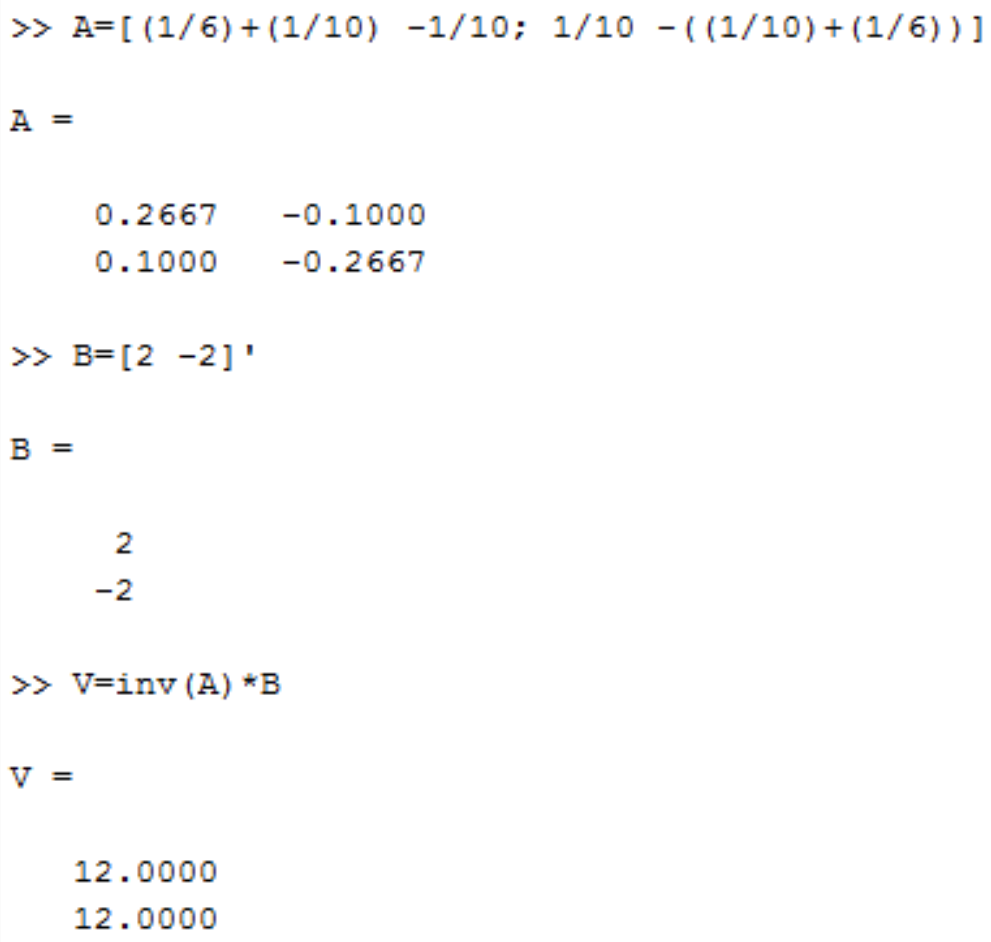

\section{Circuit Modeling}

A direct current circuit is mainly composed of resistors, current sources and voltage sources. Simulink helps simulate the circuit to compute nodal voltages. We can model the circuit in figure 3.0 to determine the nodal voltages at node 1 and node 2. Following are the modelling steps:

- Model the resistors through the Simulink block (SimPowerSystems-- $\rightarrow$ Elements-$\rightarrow$ Series RLC Branch. (Set the resistance to the given value, set $\mathrm{L}=0$ and $\mathrm{C}=\mathrm{inf}$ )

- Model the current source through the Simulink block (SimPowerSystems-- $\rightarrow$ Electrical Sources-- $\rightarrow$ Controlled Current Source)

- Model the constant for the current source through (Simulink-- $\rightarrow$ Sources-- $\rightarrow$ Constant)

- Model the voltage measurement through (SimPowerSystems-- $\rightarrow$ Measurements-$\rightarrow$ Voltage Measurement)

- Block name display can be invoked through (Simulink-- $\rightarrow$ Sinks-- $\rightarrow$ Display)

- Nodes can be assigned by invoking the neutral through (SimPowerSystems-- $\rightarrow$ Elements$--\rightarrow$ Neutral. The node number can be changed by double click on the neutral.

- Continuous power GUI must be embedded in the circuit. (SimPowerSystems-- $\rightarrow$ power GUI 
Model of the circuit for the nodal analysis to compute voltages $\mathrm{v} 1$ and $\mathrm{v} 2$ is shown in figure 4.0

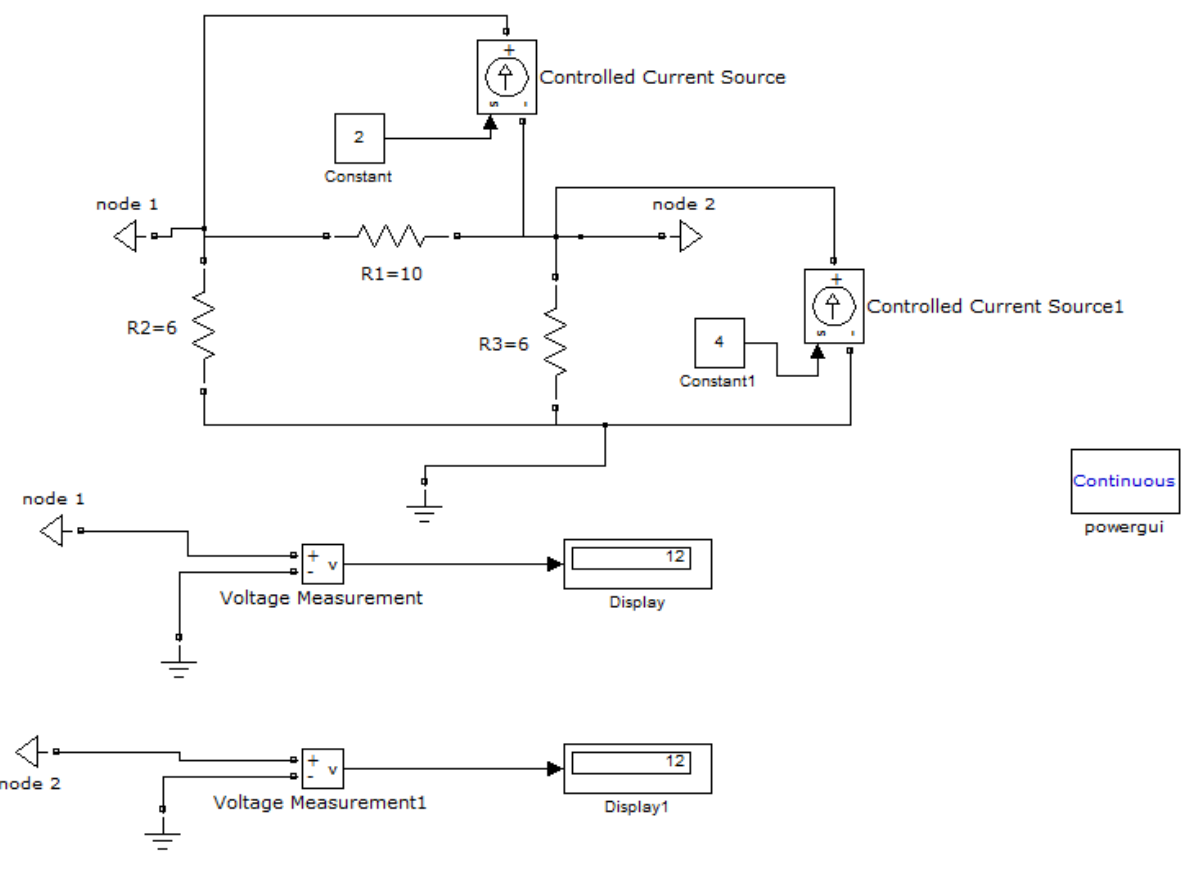

| Figure 4.0 Model of the circuit of figure 3.0 for the node voltage analysis

\section{Student Retention and Outreach:}

In fall 2013 semester, students in Electric Circuit I were surveyed to evaluate the effectiveness of introducing MATLAB and Simulink in Electric Circuit I. The statements that composed the survey are given in Table 1.0

\begin{tabular}{|l|l|}
\hline$\#$ & Question \\
\hline 1 & $\begin{array}{l}\text { Implementation of series/parallel circuits in MATLAB helped me to understand the } \\
\text { concept }\end{array}$ \\
\hline 2 & $\begin{array}{l}\text { Implementation of Nodal/Mesh analysis in MATLAB/Simulink helped to apply nodal } \\
\text { analysis in a circuit. }\end{array}$ \\
\hline 3 & $\begin{array}{l}\text { Implementation of Superpositon in MATLAB helped me to understand the basis of the } \\
\text { theorem }\end{array}$ \\
\hline 4 & Implementation of Thevenin's/Norton's Theorem helped me to understand the concept \\
\hline
\end{tabular}

Table 1.0

A five-point Likert scale was used to construct the possible responses: 1-Strongly disagree, 2Disagree, 3-Not sure, 4-Agree, and 5-Strongly agree. Participants were asked to select the response "that best describes your level of agreement" with each statement. A summary of the responses is tabulated in Table 2.0 


\begin{tabular}{|l|l|l|l|l|l|l|l|l|}
\hline Questions & SD(1) & $\mathbf{D}(\mathbf{2})$ & $\mathbf{N S ( 3 )}$ & $\mathbf{A}(\mathbf{4})$ & $\mathbf{S A}(\mathbf{5})$ & Total & Mean & $\begin{array}{l}\text { Std } \\
\text { Dev }\end{array}$ \\
\hline 1 & 0 & 0 & 0 & 11 & 5 & 16 & 4.31 & .478 \\
\hline 2 & 0 & 0 & 2 & 7 & 7 & 16 & 4.31 & .704 \\
\hline 3 & 0 & 2 & 1 & 7 & 6 & 16 & 4.06 & .997 \\
\hline 4 & 1 & 4 & 2 & 6 & 3 & 16 & 3.37 & 1.258 \\
\hline
\end{tabular}

Table 2.0

$\mathrm{SD}=$ Strongly Disagree

$\mathrm{D}=$ Disagree

NS=Not Sure

$\mathrm{A}=$ Agree

$\mathrm{SA}=$ Strongly Agree

Table 2.0 indicates that the mean ranges from 3.37 to 4.31 which strongly suggests that most of the students benefited from the inclusion of MATLAB/Simulink in Electric Circuit I course.

Retention rate was increased from $50 \%$ to $80 \%$.

Every summer the Upward Bound program hosts a summer program called Junior Engineering Technology Summer (JETS) activity which is geared towards providing, engineering experience to 9-12 grade students. In summer of 2013 the students were introduced to the following basic concepts in MATLAB:

- Entering commands and creating variables

- Analyzing vectors and matrices

- Writing functions

- Plotting

- Solving simultaneous equations

- Working with the MATLAB user interface

The Upward-Bound/JETS program has a vision to promote engineering and technology concepts and their relationship to science and math to a wide range of high school students, increasing the pool of students who will be both prepared for and interested in engineering and technology careers. Activities of the JETS program are based on the concept of hands on engineering education which has been evolving over the last few decades as an emerging field of pedagogy within engineering and technology curricula, and a focus on hands on helps to bridge the gap for high school students entering engineering and technology education. With hands on approach, the students' understanding of the material deepens by requiring them to apply the learned techniques to an open-ended task.

\section{Conclusion}

Introduction of MATLAB/Simulink in Electric Circuits I course in enhances the interest of students in problem solving and understanding the Electric Circuits I concepts leading to higher matriculation rates, increased retention, and ultimately a higher graduation rate. 
This paper emphasizes the evolution of the hands-on approach in Engineering Technology education and shows how this approach can be developed, taught, and shared with high school students.. The paper highlights the importance of outreach programs. These programs give the students and faculty the opportunity to share their understanding, and promote interest with the high school student population. The variety of these hands-on activities helps students realize the impact of math and science in the Electronics Engineering Technology environment, and their intrinsic relationships to each other. In addition, student involvement in this process promotes enhanced communication skills and develops the ability to function exceptionally well in a teambased environment.

\section{Bibliography}

1. Mohammad Nuruzzaman, "Electric Circuit Fundamentals in MATLAB and Simulink", 2007, Booksurge Publishing.

2. Robert L. Boylestad, 'Introductory Circuit Analysis", $12^{\text {th }}$ edition, Prentice Hall.

3. Charles K. Alexander and Matthew N.O.Sadiku, "Fundamentals of Electric Circuits", McGraw Hill.

4. Dorf, Richard, "Introduction to Electric Circuits', $8^{\text {th }}$ edition, John Wiley $\&$ Sons

5. Svoboda, James A.; Dorf, Richard C, "Introduction to Electric Circuits, $9^{\text {th }}$ edition, John Wiley \& Sons.

6. David M. Smith, "Engineering Computation with MATLAB", $3^{\text {rd }}$ edition, Prentice Hall.

7. Duane Hanselman; Bruce Littlefield, "Mastering MATLAB', $1^{\text {st }}$ edition, Prentice Hall.

8. David, McMahon, "MATLAB Demystified", McGraw Hill.

9. Dr. Nikunja Swain, Wanda Moses, Dr. James Allen Anderson and Cynthia T Davis, "Computational Thinking in K-12 Schools Using Hands-on Activities”, Proceedings of the ASEE Annual conference, 2013, Atlanta, Georgia.

10. Simard, C., (2009). Obstacles and Solutions for Underrepresented Minorities in Technology. www.anitaborg.org/news/research.

11. J. W. Pierre, F. K. Tuffner, "A One-Credit Hands-On Introductory Course In Electrical and Computer

12. Engineering Using a Variety of Topics Modules", IEEE Transactions on Education, vol.52, No. 2, May 2009.

13. E. Moesby, H. H. W. Johannsen, and L. Kornov, "Individual activities as an integrated part of project work: An innovative approach to project oriented and problem-based learning," World Trans. Eng. Tech. Educ., vol. 5, no. 1, pp. 11-17, 2006

14. Ruben Rojas-Oviedo, Dr. X. Cathy Qian, "Improving Retention of Undergraduate Students in Engineering through Freshman Courses", Proceedings of the ASEE Annual, Montréal, Quebec, Canada 2002.

15. Ruben Rojas-Oviedo, Dr. X. Cathy Qian, "Improving Retention of Undergraduate Students in Engineering through Freshman Courses", Proceedings of the ASEE Annual, Montréal, Quebec, Canada 2002.

16. Andrew Kline, Betsy M. Aller, and Dr. Edmund Tsang, "Improving student retention in STEM disciplines a Model that has worked", Proceedings of the ASEE Annual Conference, 2011. 